\title{
Cholesterol metabolism is a potential therapeutic target in Duchenne muscular dystrophy
}

\author{
Fatima Amor $^{1,2+}$, Ai Vu Hong ${ }^{1,2+}$, Guillaume Corre ${ }^{1,2}$, Mathilde Sanson ${ }^{1,2}$, Laurence Suel ${ }^{1,2}$, Stephanie Blaie ${ }^{1}$, Laurent \\ Servais $^{3}$, Thomas Voit ${ }^{4}$, Isabelle Richard ${ }^{1,2}$ \& David Israeli ${ }^{1,2 *}$ (DD \\ ${ }^{1}$ Généthon, Evry, France; ${ }^{2}$ Université Paris-Saclay, Univ Evry, Inserm, Genethon, Integrare research unit UMR_S951, Evry, France; ${ }^{3}$ MDUK Oxford Neuromuscular Center, \\ Department of Paediatrics, University of Oxford, UK \& Division of Child Neurology, Centre de Référence des Maladies Neuromusculaires, Department of Paediatrics, \\ University Hospital of Liège \& University of Liège, Liège, Belgium; ${ }^{4}$ NIHR Great Ormond Street Hospital Biomedical Research Centre and Great Ormond Street Institute of \\ Child Health, University College London, London, UK
}

\begin{abstract}
Background Duchenne muscular dystrophy (DMD) is a lethal muscle disease detected in approximately 1:5000 male births. DMD is caused by mutations in the DMD gene, encoding a critical protein that links the cytoskeleton and the extracellular matrix in skeletal and cardiac muscles. The primary consequence of the disrupted link between the extracellular matrix and the myofibre actin cytoskeleton is thought to involve sarcolemma destabilization, perturbation of $\mathrm{Ca}^{2+}$ homeostasis, activation of proteases, mitochondrial damage, and tissue degeneration. A recently emphasized secondary aspect of the dystrophic process is a progressive metabolic change of the dystrophic tissue; however, the mechanism and nature of the metabolic dysregulation are yet poorly understood. In this study, we characterized a molecular mechanism of metabolic perturbation in DMD.

Methods We sequenced plasma miRNA in a DMD cohort, comprising 54 DMD patients treated or not by glucocorticoid, compared with 27 healthy controls, in three groups of the ages of 4-8, 8-12, and 12-20 years. We developed an original approach for the biological interpretation of miRNA dysregulation and produced a novel hypothesis concerning metabolic perturbation in DMD. We used the mdx mouse model for DMD for the investigation of this hypothesis.

Results We identified 96 dysregulated miRNAs (adjusted $P$-value $<0.1$ ), of which 74 were up-regulated and 22 were down-regulated in DMD. We confirmed the dysregulation in DMD of Dystro-miRs, Cardio-miRs, and a large number of the DLK1-DIO3 miRNAs. We also identified numerous dysregulated miRNAs yet unreported in DMD. Bioinformatics analysis of both target and host genes for dysregulated miRNAs predicted that lipid metabolism might be a critical metabolic perturbation in DMD. Investigation of skeletal muscles of the mdx mouse uncovered dysregulation of transcription factors of cholesterol and fatty acid metabolism (SREBP-1 and SREBP-2), perturbation of the mevalonate pathway, and the accumulation of cholesterol in the dystrophic muscles. Elevated cholesterol level was also found in muscle biopsies of DMD patients. Treatment of mdx mice with Simvastatin, a cholesterol-reducing agent, normalized these perturbations and partially restored the dystrophic parameters.

Conclusions This investigation supports that cholesterol metabolism and the mevalonate pathway are potential therapeutic targets in DMD.
\end{abstract}

Keywords Duchenne muscular dystrophy; Host gene; Biological interpretation of miRNA dysregulation; SREBP-1; SREBP-2; Lipid metabolism; Cholesterol; Simvastatin; DLK1-DIO3; Glucocorticoid 


\section{Introduction}

Duchenne muscular dystrophy (DMD) is the most common inherited paediatric muscle disorder. It is an X-linked genetic progressive myopathy characterized by muscle wasting and weakness, which leads to loss of motor functions, cardiac and respiratory involvement, and premature death. ${ }^{1}$ DMD occurs at a rate of approximately 1:5000 male births and arises due to mutations in the dystrophin gene. The disease is caused by a deficiency of functional dystrophin, a critical protein component of the dystrophin-glycoprotein complex acting as a link between the cytoskeleton and the extracellular matrix in skeletal and cardiac muscles. ${ }^{2}$ The only routinely used medication for DMD patients is glucosteroid drugs, which can at best only slightly delay the progression of the disease. $^{3}$ Experimental therapeutic approaches, based on gene therapy, cell therapy, and drug discovery, are focused on the restoration of dystrophin expression. ${ }^{4}$ However, despite increasing efficiency in the restoration of dystrophin expression in recent clinical trials, only modest muscle functional improvement has been achieved. ${ }^{5,6}$ A recently emphasized aspect of the dystrophic process is a progressive metabolic change of affected muscle. ${ }^{7-11}$

Profiling miRNA can be useful for diagnosis, monitoring, and understanding mechanisms of diseases. DMD has been the subject of a large number of miRNA profiling studies. ${ }^{12-17}$ However, these studies have been hampered by one or a combination of limitations, including the use of animal models rather than human patients, the relatively small size of the studied cohorts, the detection of a small number of predefined miRNAs, or the use of miRNA profiling technologies with low sensitivities. In the present study, we overcame these limitations by profiling circulating miRNAs in the plasma of a relatively large patient cohort, which was composed of three age subgroups between the ages of 4 and 20 years old, and included both glucocorticoids (GC) treated and untreated DMD patients, as well as an age-match control group. Additionally, the profiling technology was of miRNA sequencing, maximizing the detection sensitivity of the entire spectrum of expressed miRNAs. Finally, an original bioinformatics model for the interpretation of miRNA dysregulation was employed.

Interestingly, the bioinformatics analysis of plasma miRNAs dysregulation identified lipid metabolism as the most important metabolic perturbation in DMD. In order to validate this prediction, we screened muscle biopsies of young $\mathrm{mdx}$ mice for the expression of factors that are related to lipid metabolism and identified their dysregulation as early as at the age of 5 weeks. Specifically, the mevalonate pathway that controls the synthesis of cholesterol was highly affected and cholesterol level was increased in the dystrophic muscles. A recent paper reported a positive effect of simvastatin on the dystrophic parameters and muscle function in the $\mathrm{mdx}$ mouse. Surprisingly, considering the known target of simvastatin, its effect was reported to be unrelated to blood cholesterol. ${ }^{18}$ We treated $\mathrm{mdx}$ mice with simvastatin and confirmed improved dystrophic muscle parameters. We demonstrated that components of the muscle mevalonate pathway and cholesterol levels were dysregulated in the muscle of $\mathrm{mdx}$ mouse compared with those of the healthy control and returned to normal after simvastatin treatment. In addition, the normalization of lipid metabolism correlates with improved dystrophic parameters in $\mathrm{mdx}$ dystrophic muscle. In conclusions, besides the discovery of a large number of dysregulated miRNA in the plasma in DMD patients, the present study provides new understanding of the metabolic perturbation in DMD and thus opens up new perspectives for the treatment of DMD.

\section{Methods}

\section{Ethical declaration}

The human study (DMD patients and controls) was conducted according to the principles of the declaration of Helsinki 'ethical principles for medical research' and was specifically approved by the ethical committee CPP Ile de France VI, on 20 July 2010, and the Comité d'Ethique (412) du CHR La Citadelle (Liège, Belgium) 26 January 2011.

\section{Human patients and cohort composition}

Duchenne muscular dystrophy patients were admitted from 10 European medical centres, two of them from Belgium $(n=35)$, one from Romania $(n=16)$, and seven from France $(n=49)$. Healthy control patients were admitted from the same medical centres, Belgium ( $n=28)$, Romania $(n=45)$, and France $(n=50)$. Patients were divided into three age groups $4-8,8-12$, and $8-20$ years old. In the youngest age group, the same donors contributed the GC-treated and untreated samples, with untreated samples that were obtained before, and treated samples after their first GC treatment, at an interval of less than 6 months. GC-treated and untreated samples for the age groups of 8-12 and 1220 years old were obtained from distinct DMD patients. Human skeletal muscle tissues were obtained from the Myobank, the tissue bank of the Association Francaise contre les Myopathies (AFM). Open skeletal muscle biopsies were performed after informed consent, according to the Declaration of Helsinki. Muscle biopsies included in this study were derived from the paravertebral (two controls and two DMD), gluteus, and the tensor fasciae latae (one each control and one DMD) from the group aged 8-12 (DMD) and 12-20 (control) years old. 


\section{Enrichment analysis of microRNA targets}

The miRNA-target gene enrichment analysis was performed with the web-based mirPath v. $3^{19}$ http://snf-515788.vm. okeanos.grnet.gr/, based on predicted miRNA-mRNA interaction in human.

\section{MicroRNA host genes analysis}

For this analysis, we considered all the dysregulated miRNAs $(P \leq 0.05)$ in DMD patients aged $4-12$ years old, compared with their age-related healthy controls (Supporting Information, Table S2). MicroRNA host genes were retrieved from the miRIAD database ${ }^{20}$ and were manually validated on the Ensembl human genome browser GRCh38.p7. We considered all miRNAs embedded on the sense strand of introns and exons of protein-coding genes. We assigned to the hostgenes the fold change and $P$-values of their embedded miRNAs. When both $-3 p$ and $-5 p$ isoforms of the same pre-miRNA were dysregulated, their host-genes were considered only once, with the lower $P$-value miRNA isoform. Similarly, host genes for a number of different miRNAs in a miRNA cluster were considered only once, with the highest dysregulated miRNA (according to $P$-value). In miRNAs hosted on more than one host-gene, both host-genes were considered. The table of host-genes with their assigned dysregulation values is in Supporting Information, Table S8. Host genes data were assigned to the core analysis of Ingenuity ${ }^{\circledR}$ Pathway Analysis (IPA ${ }^{\circledR}$, QIAGEN Redwood City, www.qiagen.com/ ingenuity). Pathway enrichment of the gene list was also performed by ReactomePA and ClusterProfiler R/Bioconductor packages. $^{21}$

For the procedures of blood collection, RNA extraction, choice of samples for the miRNA profiling, miRNA sequencing, in vivo mice experiments, RNA expression analysis, western blotting, immunofluorescence, and measurement of plasma free cholesterol, creatine kinase, and myomesin-3 are provided in the extended material and methods section in the supporting information.

\section{Results}

\section{Duchenne muscular dystrophy patient characterization and cohort composition}

To perform miRNA profiling in the serum of DMD patients, we collected samples of 100 DMD patients and 123 healthy controls from 10 European medical centres (Supporting Information, Table S1). The study was approved by central and local Institutional Review Board (IRB) and recorded on ClinicalTrials.gov (Identifier: NCT01380964). All patients or parents for minors signed informed consent. Following RNA extraction from the plasma and quality control validation, we selected 81 RNA samples and constituted nine groups of nine patients. The groups consist of DMD patients, either untreated (DMD-UT) or receiving treatment with glucocorticoid (DMD-T) and healthy controls, in three age categories of 4-8, $8-12$, or $12-20$ years old (Figure $1 A$ ). The mean age of disease onset of the corresponding cohort was 3 years of age. Walking capacity was preserved in all patients of the first age group, while three patients of the second group $(33 \%$ of untreated DMD), and all but two patients of the (GC-treated) 12-20 years old had lost their ability to walk (Supporting Information, Table S2). Overall, the mean age of loss of walking ability was 9.6 years. Additional clinical and functional characterization of the patients is shown in Supporting Information, Table S2, and the spectrum of dystrophin mutations present in the patients is depicted in Figure $1 B$.

\section{MicroRNA dysregulation in Duchenne muscular dystrophy is age-dependent}

After size selection, the 81 RNA samples were all individually sequenced using the Illumina technique. All mapped reads were matched to the hg19 (GRCh38) human genome assembly and were assigned to RNA classes, including rRNA, snoRNA, piRNA, tRNA, and miRNA (miRbase v20, June $2013^{22}$ ) (Figure $2 A$ ). We obtained 9.37, 3.68, and 2.81 million sequences on average per studied sample for total high quality (high quality reads), human genome mapped (mapped reads), and mature miRNA sequences, respectively (Figure $2 B$ ). A principal component analysis ( $P C A$ ) was used for the identification of overall miRNA profile dysregulation between the different cohort subgroups. PCA failed to separate DMD from control samples (Figure $2 C$, upper left). In contrast, PCA primary and secondary components separated the DMD (orange dots) from the control (blue dots) of the 4-8 and $8-12$ age groups (Figure $2 C$, upper middle and right, respectively) but not of the 12-20 age group (bottom left). In the 4-12 years old group (combined $4-8$ and 8-12 groups), DMD patients were separated from healthy controls (bottom middle). However, PCA failed to separate GC-treated from untreated DMD patients (bottom right). Of note, PCA analysis failed to separate miRNA profiles according country of origin (France, Belgium, and Rumania), in the 4-12 years old DMD patients, excluding hypothesis of strong impact of country of origin (data not shown). Of interest, no significant correlation was found in DMD between the levels of any single circulating miRNA to patients' body mass index (BMI) (data not shown). These results indicate a robust disease effect, but not such robust GC treatment or country of origin effects, on circulating miRNA in DMD. This effect is 

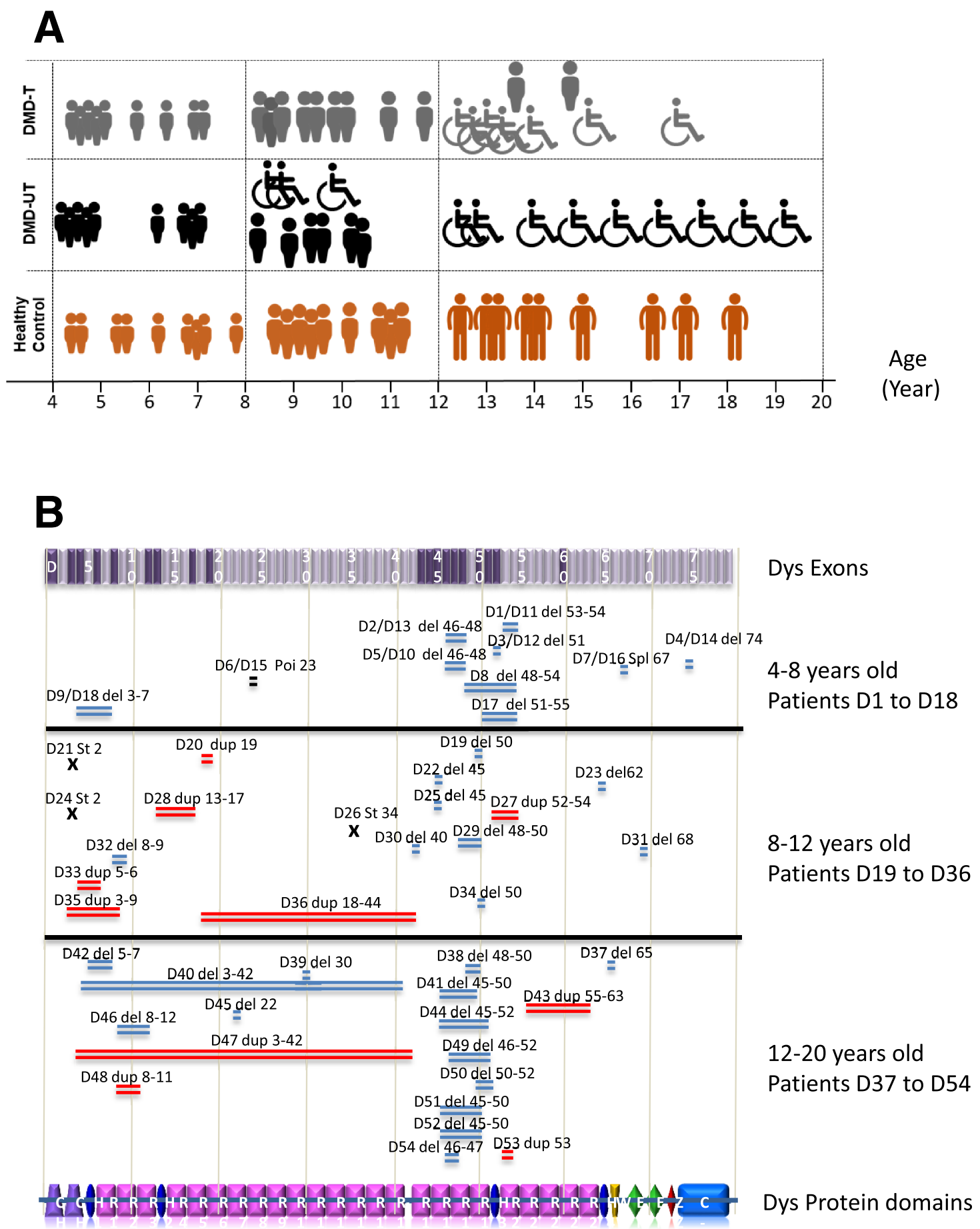

\section{$=$ Deletion $\quad=$ Duplication $\quad=$ Point mut. $\quad x$ Stop mut. $\quad$ Spl Splice mut.}

Figure 1 DMD cohort characterization. (A) cohort subgroups and age composition. DMD patients and healthy controls were classified into three age groups of 4-8, 8-12 and, 12-20 years old. DMD patients were glucocorticoid treated (DMD T) or untreated (DMD UT). The figurine symbols represent the ages of individual patients on the horizontal axis, black for untreated DMD, grey figures for treated DMD, and brown figures for healthy controls. Treated and untreated DMD of the 4-8 group of age are the same patients before and after GC treatment (except one patient). (B) A graphical presentation of the spectrum of dystrophin mutations by age group. Dystrophin's gene 79 exons and protein domains are presented on respectively the upper and lower vertical bands. Patients of the 4-8 age years old group are represented twice in the cohort (with the exceptions of D8 and D17), before and after glucocorticoid treatment, by samples D1 to D9 and D10 to D18, respectively. Del (blue) = deletion; dup (red) = duplication; St (black) $=$ stop codon mutation; poi (in black) $=$ point mutation . 
A

81 plasma samples

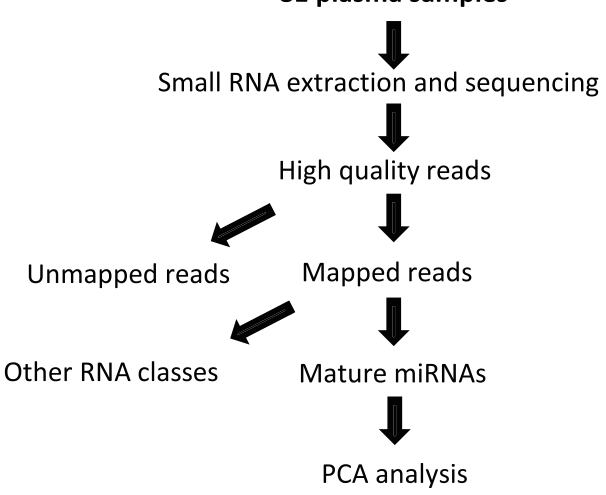

B

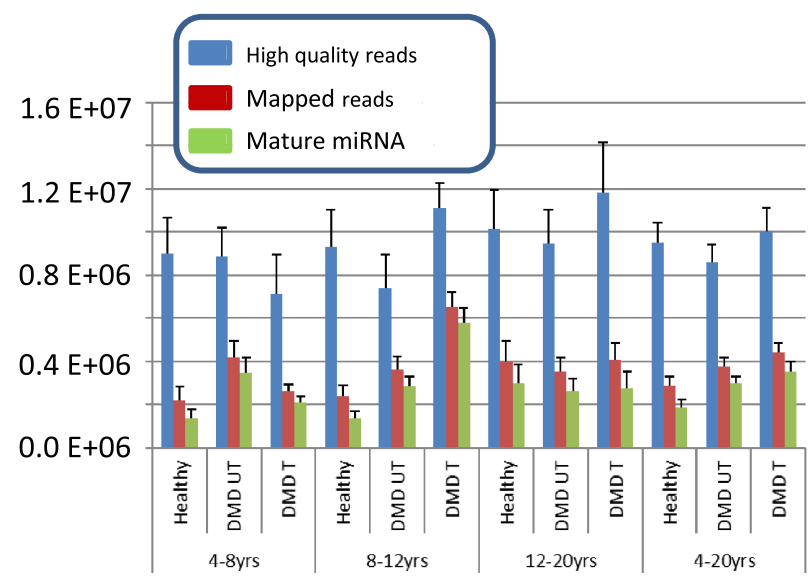

4-20 YO

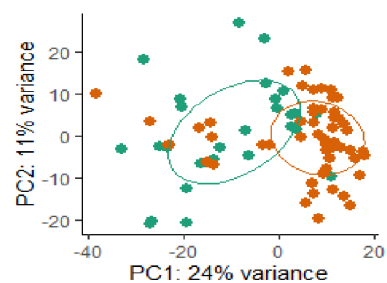

12-20 YO

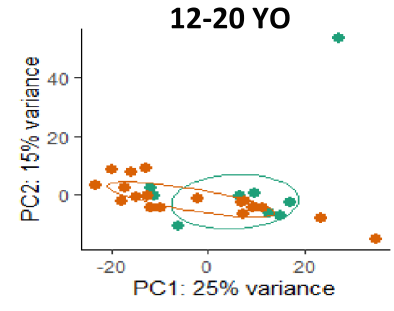

4-8 YO

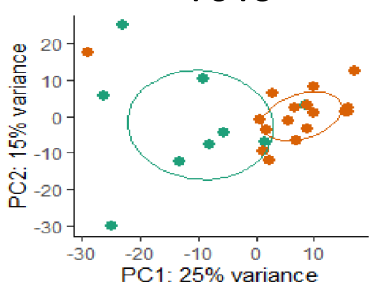

4-12 YO

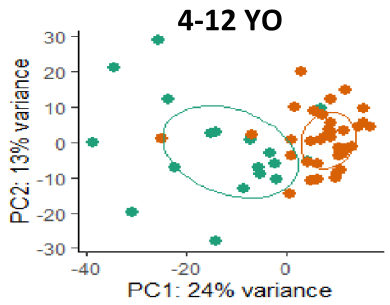

8-12 YO

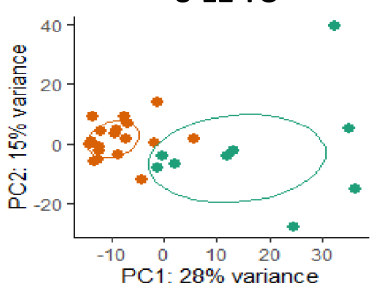

- Control

- DMD

Figure 2 Characterization of circulating RNA by high throughput sequencing. (A) schematic presentation of plasma sample processing. Small RNA high quality reads (high quality reads) were mapped on the human genome (mapped reads). Mapped reads were classified to miRNA and other small RNA classes. (B) Graphical presentation of high quality reads (blue), mapped reads (red), and miRNA (green) in the cohort subgroups are presented as average \pm SEM. (C) PCA analysis of cohort segregation according to miRNA expression in DMD (orange dots) and healthy control (green dots) by age groups (panels 1 to 5) and treated (red dots) versus untreated (black dots) DMD patients (panel bottom right). 
age-dependent as it is no longer observed beyond the age of 12 .

\section{Differentially expressed plasmatic microRNAs in Duchenne muscular dystrophy patients}

The aforementioned data indicate that the overall miRNA dysregulation in DMD patients is drastically reduced beyond the age of 12. In accordance, upon stratification on age and treatment, we noticed that the largest set of dysregulated miRNAs was identified by comparing the combined GCtreated and untreated 4-12 years old patient group to their age-related healthy controls (Supporting Information, Figure S1), including 65 up-regulated and 25 down-regulated miRNAs (adjusted $P$-value $<0.1$ ) in DMD patients (Supporting Information, Tables S3 and S4, and Figure $3 A$ ). Among the dysregulated miRNAs, we validated the dysregulation in DMD of the dystro-miRs, ${ }^{12-15}$ the heart-enriched cardiomiRs, and a large number of miRNAs belonging to the DLK1-DIO3 cluster. $^{15}$ Of the newly identified dysregulated miRNAs, we noticed in particular a large number of the Let-7 family members, the entire miR-320 family, and many miRNAs, which are known modulators of diverse biological functions in skeletal and cardiac muscles, including miR-128, ${ }^{23}$ miR-199a, ${ }^{24}$ miR-223, ${ }^{25}$ miR-486, ${ }^{26}$ and members of the miR- $29^{27}$ and miR-30 28 families. Of interest, six of the dysregulated miRNAs were reported to target dystrophin expression in the dystrophic muscle, including the upregulated miR-494, miR-223, miR-103a, Let-7d and Let-7a, and the down-regulated miR-320a. ${ }^{29}$ The proximity between miRNA class members on the Volcano plot suggested their similar dysregulation. Indeed, highly significant expression correlations were identified among miRNA members within the distinct miRNA classes (Supporting Information, Figure S2), supporting their coordinated dysregulation. The dysregulation pattern of a selected number of miRNAs are presented graphically (Figure 3), including the up-regulated miR-206, miR-208a-3p, miR-128-3p, miR-199a-3p, and miR369-5p (Figure 3B), the down-regulated miR-342-3p, miR320a, miR-361-3p, miR-29b-3p, and miR-30e-5p (Figure $3 C)$, and the GC-responsive miR-223-3p, miR-379-5p, miR$27 b-5 p$, and Let-7d-5p (Figure 3D). To analyse the glucocorticoid response, we compared miRNA expression before and after glucocorticoid treatment in the 4-12 age group DMD patients and identified 11 miRNAs, which were dysregulated in untreated DMD patients compared with healthy controls, and for which the expression was significantly changed by the glucocorticoid treatment. The expression of nine of these miRNAs shifted toward normalization by the GC-treatment. Of note, miR-27b-5p, let-7i-5p, and miR-379-5p were no longer dysregulated in the group of treated DMD versus healthy control (Supporting
Information, Table S5). Similarly, miR-27b-5p and miR-379$5 p$ were not statistically different from healthy controls when all DMD patients (treated + untreated) are grouped together.

\section{Bioinformatics interpretation of miRNA dysregulation}

Investigation of predicted target genes for dysregulated miRNAs

MicroRNAs control the expression and/or stability of target genes, and consequently, the biological functions of miRNAs are mediated by their targets. A common method for investigating miRNAs functions consists of identifying signalling pathways and cellular functions of these target genes. To identify pathways that dysregulated miRNAs in DMD might control, we used the KEGG function of DIANA TOOLS miRPath $\mathrm{V}^{1}{ }^{19}$ considering all upregulated and downregulated miRNAs (adjusted $P$-value $<0.1$ ) of $4-12$ years old DMD compared with age-matched controls. The analysis identified 20 distinct significantly enriched ( $P$-value $<0.05$ ) pathways, including pathways already suspected of participating in the DMD disease such as extra-cellular matrix (ECM)-receptor interaction, fatty acid biosynthesis, focal adhesion, PI3K-AKT signalling, and Foxo signalling (Supporting Information, Table S6).

Investigation of host-genes for dysregulated microRNAs in Duchenne muscular dystrophy

We also attempted to develop a complementary procedure for the biological interpretation of miRNA dysregulation. More than half of human miRNAs are embedded within introns and exons of protein-coding genes. ${ }^{20}$ The embedded miRNAs are often co-expressed with their host genes and can regulate their expression and activity, ${ }^{30-32}$ which support functional relations between the miRNA, host-gene, target genes, and biological activity. Indeed, we found that 12 of the identified dysregulated miRNAs were embedded in seven host genes that are known to be dysregulated in DMD models and patients (Supporting Information, Table S7). Accordingly, we explored the possibility that analysis of host genes for dysregulated miRNAs may provide an insight into functional causes and consequences of miRNA dysregulation. The Ingenuity pathway analysis (IPA) package (Qiagenbioinformatics) was used for the algorithmic prediction of host-gene networks, assigning the fold change and $P$-value of their embedded miRNAs (Supporting Information, Table S8). Three networks were identified, all of which including the terms Lipid Metabolism and Small Molecule Biochemistry (Table 1). Of interest, the three networks integrate a number of biomolecules that are known to be involved in DMD pathophysiology, some of which are recognized as therapeutic targets, supporting the relevance of the analysis. It included, in 

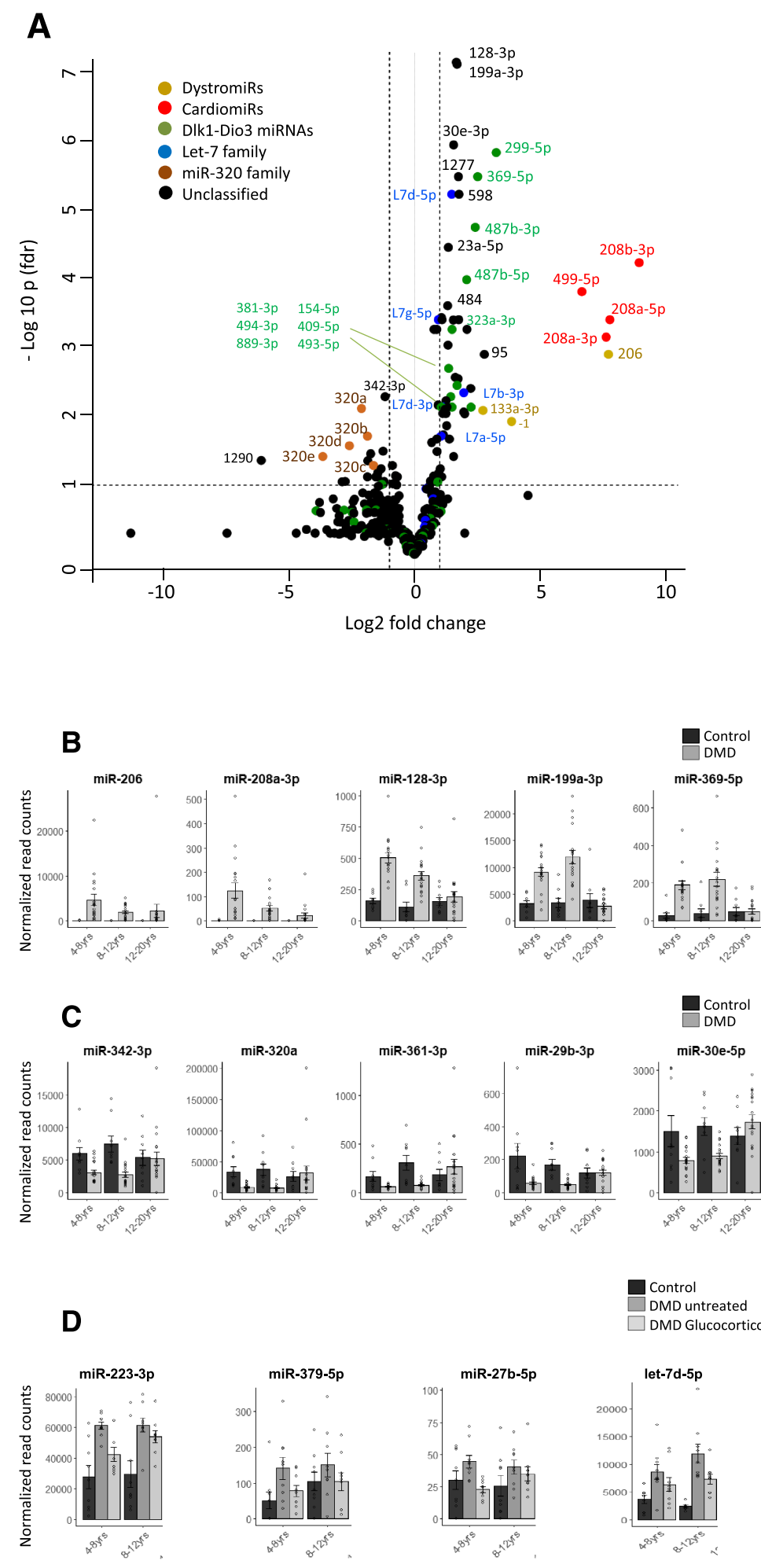

Figure 3 Plasma miRNA profiling in the DMD cohort. (A) a volcano plot of miRNA dysregulation in the plasma of 4-12 years old DMD patients (treated and untreated together, $n=36)$ versus healthy control $(n=18)$. Up-regulated miRNA in DMD are on the right side and down-regulated on the left side of the threshold lines $(|F C| \geq 1.5)$. MiRNAs above the horizontal line are differentially expressed with adjusted $p<0.1)$. DystromiRs in yellow, cardiomiRs in red, DLK1-DIO3 miRs in green, Let-7 family miR in blue, miR-320 family in brown, unclassified miR in black. (B) A graphical presentation of miRNA up-regulated in DMD. (C) A graphical presentation of miRNA down-regulated in DMD. (D) Glucocorticoid responsive miRNAs in DMD. Each dot represents one patient, $n=9$, error bar $=$ SEM. 
Table 1 Network analysis of host genes for dysregulated miRNAs

\begin{tabular}{llccc}
\hline & \multicolumn{1}{c}{ Network } & Score & $\begin{array}{c}\text { Number focus } \\
\text { molecules }\end{array}$ & $\begin{array}{c}\text { Most connected } \\
\text { molecule }\end{array}$ \\
\hline 1 & Lipid metabolism, small molecule biochemistry molecular transport & 1 e-64 & 31 & SREBF1 \\
2 & Lipid metabolism, small molecule biochemistry, molecular transport & 1 e-41 & 22 & $\begin{array}{l}\text { Beta estradiol } \\
\text { (oestrogen) }\end{array}$ \\
3 & $\begin{array}{l}\text { Lipid metabolism, small molecule biochemistry, dermatological } \\
\text { diseases, and conditions }\end{array}$ & 1 e-32 & 18 & HNFA4 \\
4 & Lipid metabolism, molecular transport & & SREBF1 &
\end{tabular}

A pathway analysis was used for the construction of gene networks, using the core analysis of ingenuity ${ }^{\circledR}$ pathway analysis (IPA) with a threshold of 70 molecule per network. The constructed networks are composed of host-genes for dysregulated miRNAs $(P<0.05)$ in the plasma of the 4-12 years old DMD patients. The $P$ value denotes the 'probability' of the coincidental construction of a network out of its components. The focus molecule (3rd column) denotes the number of the network's principal molecules, and the identity of the most connected molecule of each network is provided in the 4th column. The 4th network was obtained by merging all networks function of IPA algorithm.

network-1, insulin, MAPK, Erk1/2, PI3K and Akt, all members of the canonical muscle hypertrophy pathway, NF-KB, H3/H4 histones, PDGF and PDGFR, Hox9A, and the mediator complex. The SREBP-1 gene was identified as the most connected in the first and largest network (Network 1, Figure 4A). Network 2 includes cholesterol, ${ }^{18}$ EGFR, ${ }^{33} \alpha$ - and $\beta$-estradiol, and their oestrogen receptor. ${ }^{34}$ Beta-estradiol was the most connected molecule in this network (Network 2, Figure 4B). Network 3 includes TGF-beta and the hepatocyte nuclear factor HNF4a, which is its most connected molecule (Network 3, Figure $4 C$ ). Finally, upon integrating the three networks using the IPA software, the SREBP-1 molecule was the most connected molecule of the combined network (Figure 4D). This basic helix-loop-helix transcription factor, as well as its family member SREBP-2, can bind specific sterol regulatory element DNA sequences to up-regulate the synthesis of enzymes involved in sterol biosynthesis, thus are master regulators of lipid metabolism. ${ }^{35}$

In a complementary approach, the same 71 host genes (Supporting Information, Table S8) were subjected to a gene ontology (GO) enrichment analysis using the ReactomePA. Particular enrichment was identified for terms that are related to lipid metabolism, including the activation of gene expression by SREBP (at the upper position by $P$-value), regulation of lipid metabolism by peroxisome proliferator activated receptor alpha (PPARA), COPI mediated transport, regulation of cholesterol biosynthesis by SREBP and metabolism of steroids (Figure $4 E$ ). Considering that a large proportion of host genes are involved in lipid metabolism, the ClusterProfiler $\mathrm{R}$ package was used on a sub-selection of the host genes which are known to participate in lipid metabolism. This analysis suggested the involvement of this network in the functions of transport to Golgi, PPARA transcriptional activity and regulation of lipid metabolism, regulation of cholesterol synthesis, COPI mediated anterograde transport, metabolism of fat-soluble vitamins (vitamin D) and of steroids (Figure 4F). Thus, two different bioinformatics analyses identified the SREBP-dependent transcription program and the metabolism of lipid and cholesterol in the centre of a network of host genes for dysregulated miRNAs in DMD.

Evidence for lipid metabolism dysregulation in the $\mathrm{mdx}$ model

The miRNA-host gene analysis predicted the dysregulation of the SREBP pathway in DMD. To investigate the relevance of this prediction, we quantified the expression level of lipid metabolism components, with a focus on the SREBP pathway in the mdx mouse model (Figure 5A). In addition to SREBP-1 and SREBP-2, this analysis included the SREBP upstream regulator, SCAP, ${ }^{36}$ the SREBP-1 target gene, FASN, and the SREBP-2 target genes, HMGCR and LDLR. ${ }^{37}$ In 5-week-old mdx mice, four and three (out of the six) transcripts were significantly up-regulated in gastrocnemius (GA) and diaphragm, respectively (Figure $5 B, C$ ). At the protein level, FASN, HMGCR, SREBP-1 and SREBP-2 were up-regulated in GA (Figure 5D,E), whereas the FASN, HMGCR, SCAP, and SREBP-2 were significantly up-regulated in diaphragm (Figure $5 F, G)$. Thus, it indicated the up-regulation of both SREBP-1 and SREBP-2 expressions and activities in the dystrophic muscles.

\section{Simvastatin alleviated the dystrophic phenotype and partly normalized muscle cholesterol content in $\mathrm{mdx}$ mouse}

A positive effect of the cholesterol synthesis inhibitor simvastatin on the dystrophic muscle was previously reported in the mdx mouse model. ${ }^{18}$ The observed beneficial effect was attributed to the reduction of oxidative stress, fibrosis, and inflammation. Interestingly, a recent study reported an abnormally higher cholesterol content in muscles of DMD patients. ${ }^{11}$ Therefore, we aimed at validating the beneficial effect of simvastatin in the $\mathrm{mdx}$ mouse and its possible relation with modulation of muscle cholesterol. Orally administrated simvastatin during a 3-week period significantly reduced the level of serum myomesin-3, a sensitive serum biomarker of 

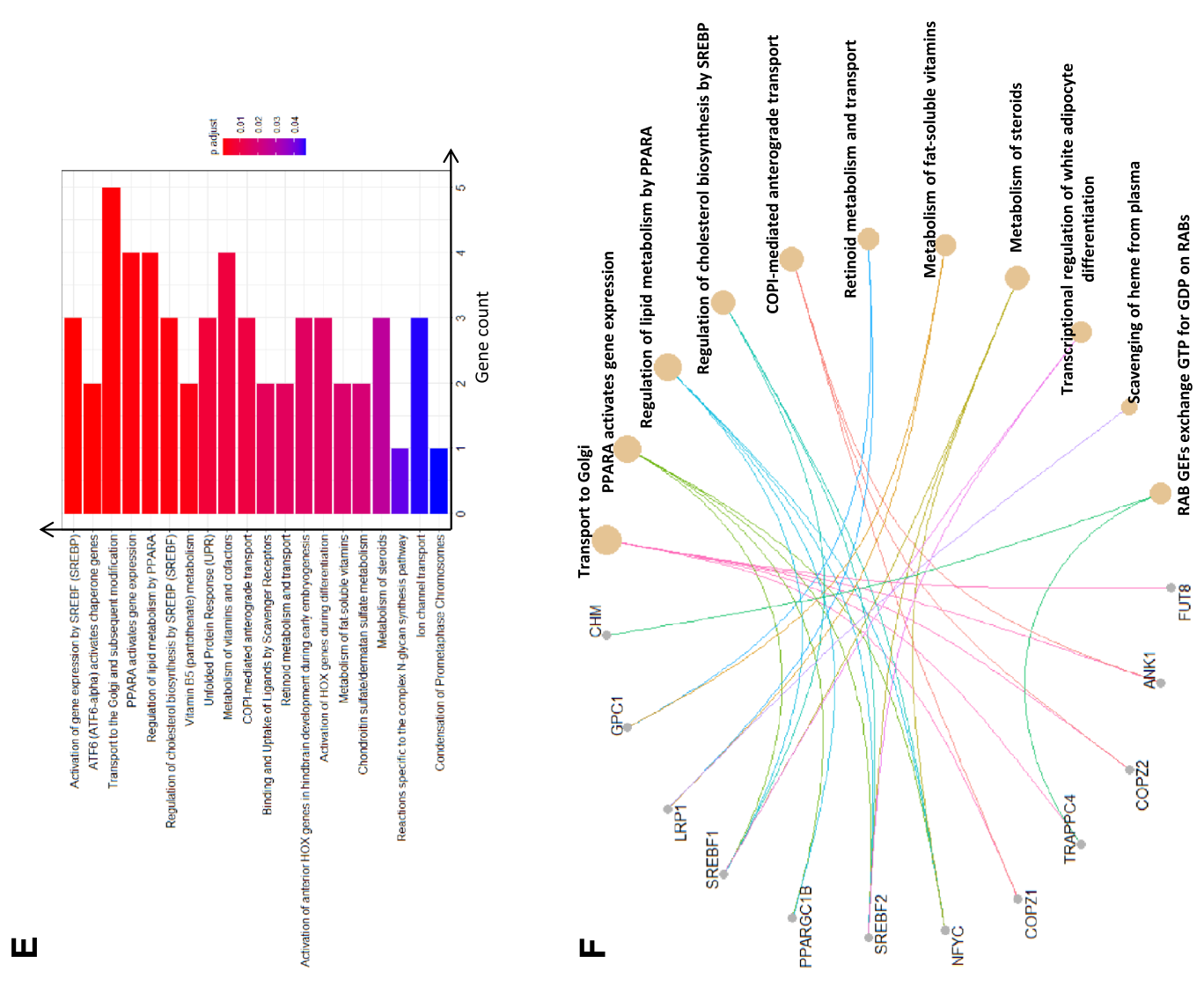

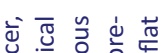

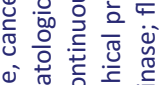

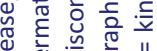

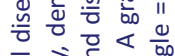

厄)

品

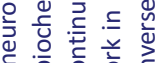

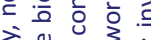

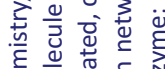

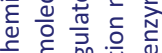

응

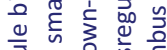

चै हो 흥 है

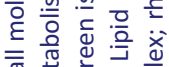

हू है

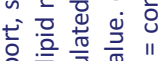

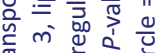

要䓂

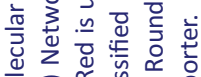

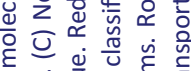

हे

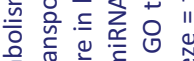

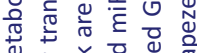

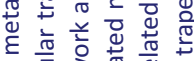

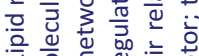

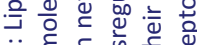

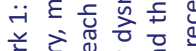

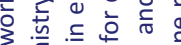

हैं

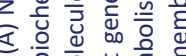

๙்

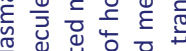

응 행 "을 "1

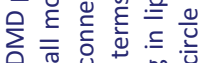

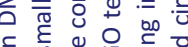

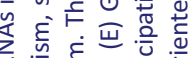

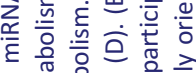

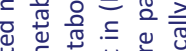

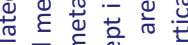

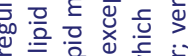

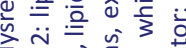

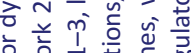

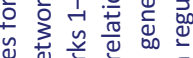

这之衣获。

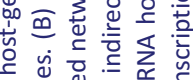

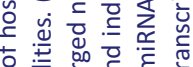

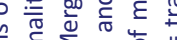

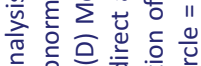

元

둥

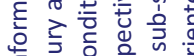

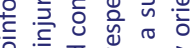

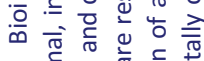

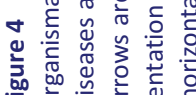


A

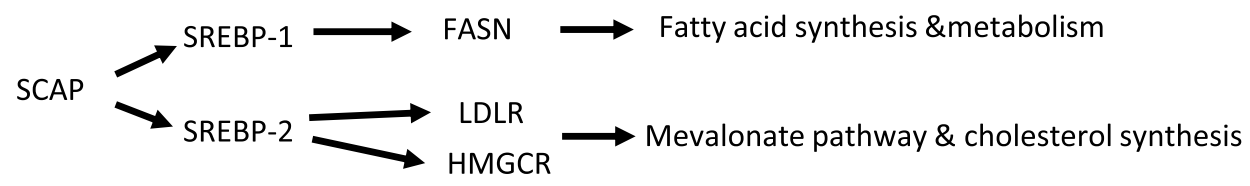

B
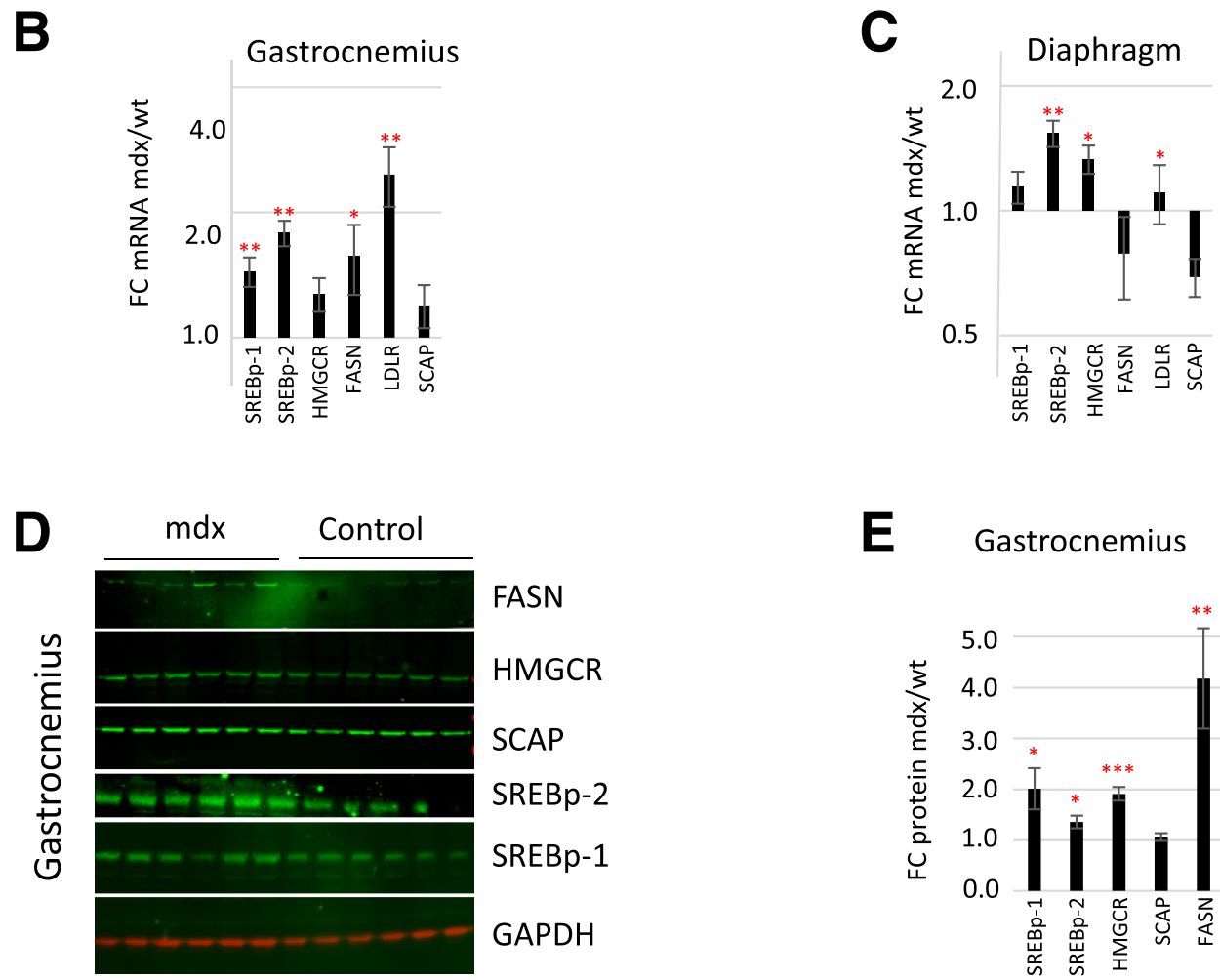

FASN

E

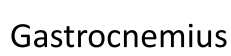

HMGCR

SCAP

SREBp-2

SREBp-1

GAPDH
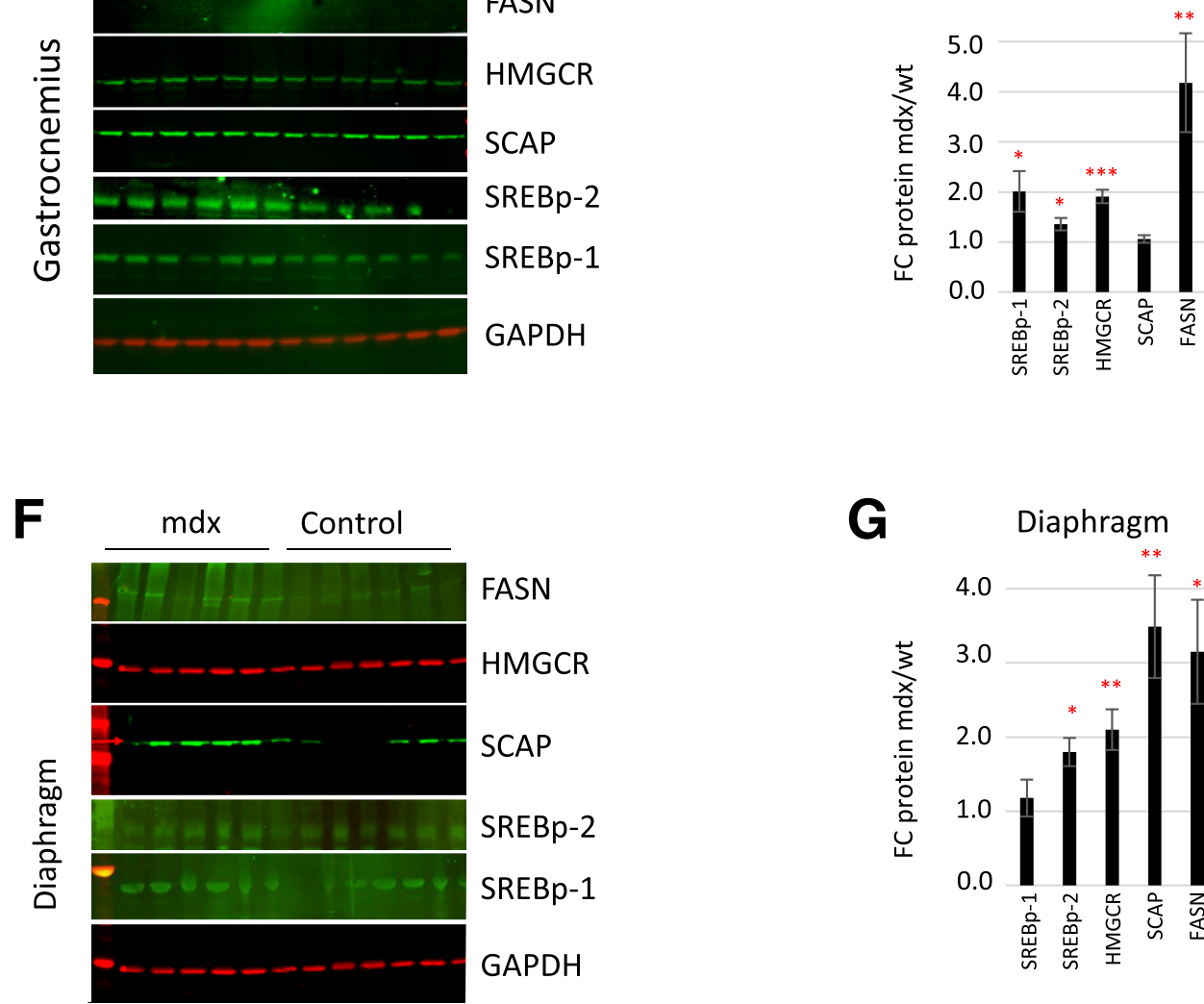

FASN

HMGCR

SCAP

SREBp-2

SREBp-1

GAPDH

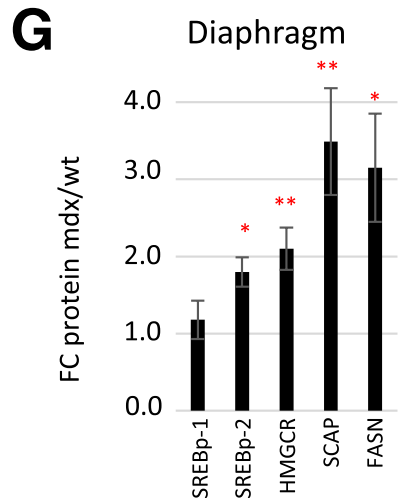

Figure 5 SREBP pathway in the $m d x$ muscle. (A) A schematic presentation of the components in the SREBP pathway (see text for details). (B, C) Fold change SREBP pathway transcripts in the gastrocnemius (B) and the diaphragm (C) in the mdx versus healthy control mouse. (D-G) A western blot analysis of SREBP pathway protein expression in the gastrocnemius (D) and its graphical quantification (E), and of the diaphragm muscle (F) and its graphical quantification (G). SREBP-1 = sterol regulatory binding element 1; SREBP-2 = sterol binding regulatory element 2; HMGCR = HMG-CoA reductase; FASN = fatty acid synthase; LDLR = low density lipoprotein receptor; SCAP=SREBP cleavage-activating protein. 


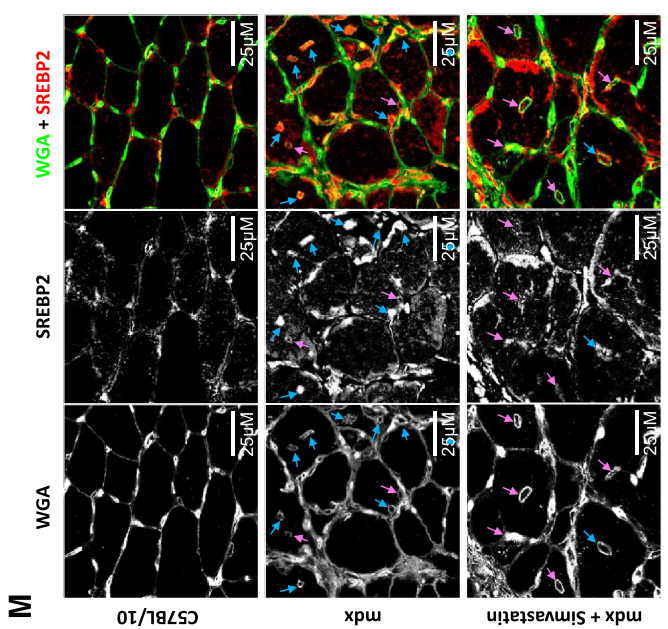

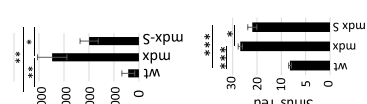

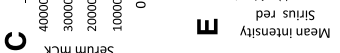

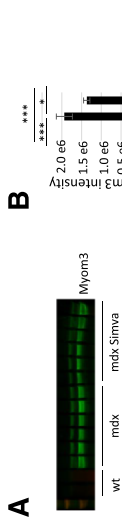

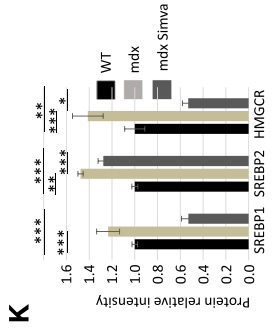

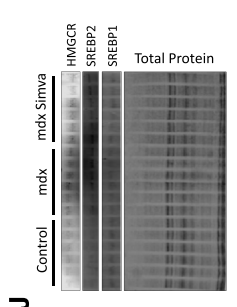

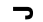
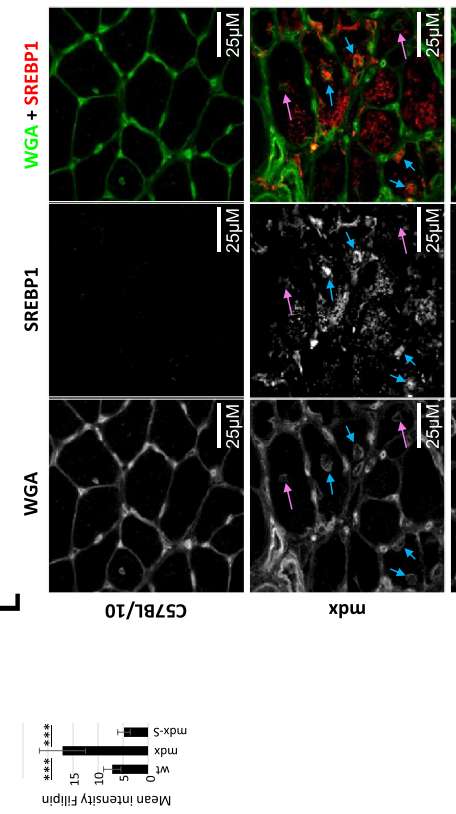

工
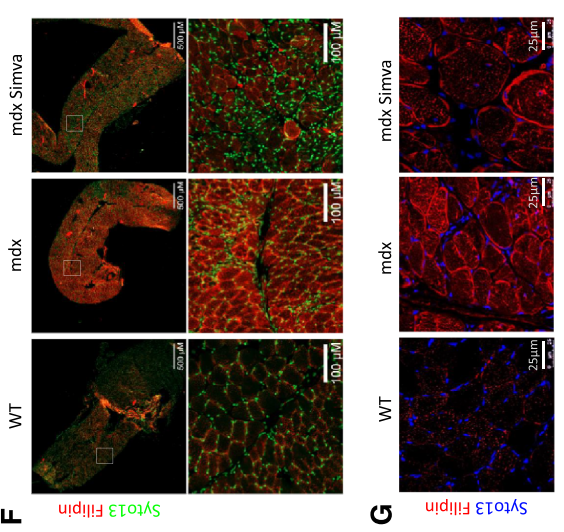

ᄂ.

v
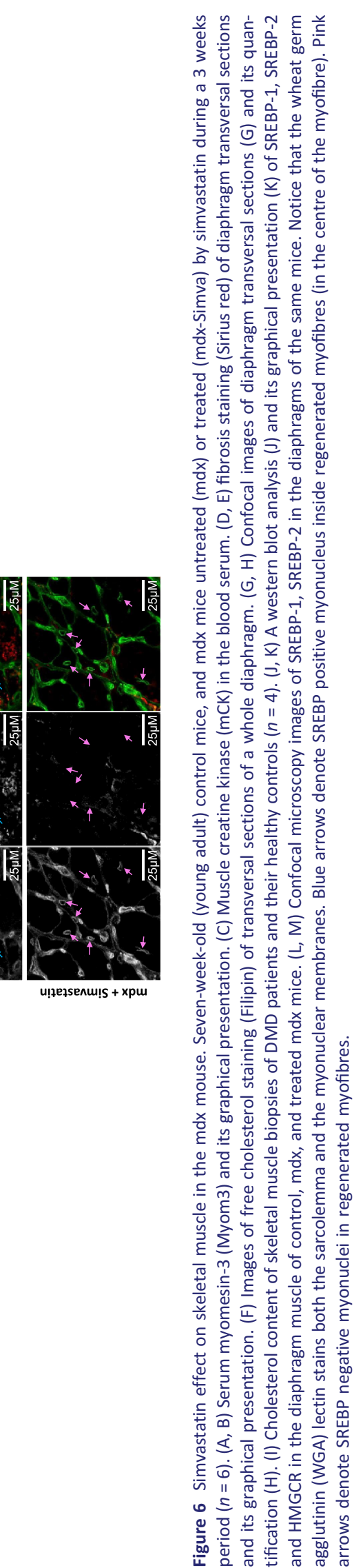

Journal of Cachexia, Sarcopenia and Muscle 2021; 12: 677-693 
muscular dystrophy ${ }^{38}$ (Figure 6A,B). The level of mCK was reduced as well in the simvastatin-treated mdx mice (Figure $6 C$ ). Histological analysis of the diaphragm muscle revealed significantly reduced fibrosis in treated mdx mice (Figure $6 D, E$ ).

The serum cholesterol level was not reduced by simvastatin (Supporting Information, Figure S3), in agreement with the observation made in a previous study. ${ }^{18}$ We sought to investigate the consequences of simvastatin treatment at the level of the muscle tissue. Filipin is a naturally fluorescent polyene antibiotic that binds to free but not esterified sterols and is useful for detecting free cholesterol in biological samples. The specificity of filipin staining to cholesterol was demonstrated by treating $\mathrm{C} 2 \mathrm{C} 12$ cells with the cholesteroltrafficking inhibitor U18666A (Supporting Information, Figure S4). Importantly, filipin staining of transversal sections confirmed increased free cholesterol content in the diaphragm of 10-weeks-old mdx mice compared to their wild-type controls (Figure 6F). Quantification of confocal images of the same sections revealed over two-fold $(P<0.001)$ increased cholesterol expression in the in mdx compared to wild-type controls, and a partial normalization of cholesterol expression in the simvastatin treated group $(P<0.001$ ) (Figure $6 G, H)$. Similar reduced cholesterol content in treated $\mathrm{mdx}$ mice were observed also in the GA and TA muscles (Supporting Information, Figure S5). Finally, the quantification of the free cholesterol content in muscle biopsies in a small cohort of human DMD patients $(n=4)$ confirmed approximately two-fold $(P=0.035)$ increased free cholesterol in DMD muscles compared to controls (Figure 6I). A western blot analysis demonstrated that simvastatin treatment normalized the expression of SREBP-1, SREBP-2, and HMGCR in the diaphragm of the $\mathrm{mdx}$ mouse (Figure $6 J, K$ ). Finally, we employed confocal microscopy for the investigation of SREBP-1 and SREBP-2 expression in transversal sections of the diaphragm. Higher levels of both proteins were observed in $\mathrm{mdx}$ compared to the wild-type control, and this expression was reversed by simvastatin (Figure 6L,M). Importantly, we noticed the expression of SREBP-1 (Figure 6L) and a more clearly high expression of SREBP-2 (Figure 6M and Supporting Information, Figure S6) inside myonucleus in the $\mathrm{mdx}$ mice. The majority of the SREBP-1 and SREBP-2 positive myonucleus was in a central position in the myofibres, indicating regenerated myofibres. Of particular interest, the expression of SREBP-1 and SREBP-2 was only rarely detected in the nuclei of regenerated myofibres of the simvastatin-treated $\mathrm{mdx}$ mice. It has been suggested that a positive effect of simvastatin treatment on the dystrophic phenotype in the mdx mouse may result from the capacity of simvastatin to reduced oxidative stress, indicated by the down-regulation of Nox2, and to activate the autophagic flux, indicated by the increased expression of the lipidated form of LC3 (LC3b). ${ }^{18} \mathrm{~A}$ western blot analysis confirmed the up-regulation of Nox2, LC3a (cytoplasmic form of LC3), and the LC3b proteins in the 10-week old mdx mice's diaphragm with, however, no detected effect of the simvastatin on their expressions (Supporting Information, Figure S7). These data supported that in young adult mdx mice, the oxidative stress and autophagy pathways are not the major targets of the observed positive effect of simvastatin. Taken together, these results indicate that (i) muscles of the $\mathrm{mdx}$ mouse are characterized by the up-regulation of the transcriptionally active nuclear forms of SREBP-1 and SREBP-2 in the centrally positioned myonucleus of regenerating myofibres. (ii) Muscle fibres of the mdx mouse are characterized by increased cholesterol content, and (iii) simvastatin treated $\mathrm{mdx}$ mice presented improved dystrophic phenotype in correlation with normalized muscle SREBP-1 and SERBP-2 expression and cholesterol content.

\section{Discussion}

In previous investigations, we profiled circulating miRNA in the serum of animal models for DMD. ${ }^{14,15,39}$ Following these studies, we are reporting the profiling of miRNA in the plasma of DMD patients. We validated and confirmed the dysregulation of many of the previously identified dysregulated circulating miRNAs in muscular dystrophy. In addition, we are reporting many newly identified dysregulated miRNAs. An original bioinformatics approach was employed, based both on target and host genes for dysregulated miRNAs. This analysis predicted a central role for the perturbation of lipid metabolism in DMD and particularly of the SREBP/mevalonate/cholesterol synthesis pathway. Analysis of skeletal muscle biopsies of the mdx mouse confirmed dysregulation of the SREBP pathway and increased cholesterol content. Treatment of $\mathrm{mdx}$ mice with simvastatin partially normalized the SREBP pathway, reduced cholesterol accumulation in the dystrophic muscles, and improved dystrophic parameters of the treated mice.

\section{The Duchenne muscular dystrophy cohort and microRNA dysregulation}

To our knowledge, this is the first study to report global miRNA profiling in the plasma of DMD patients over a large age range, including GC-treated versus untreated patients. The overall miRNA dysregulation decreased in subjects above the age of 12. Thus, this age-dependent dysregulation pattern, which was shown previously with the $\mathrm{mCK}$ and the myomiRs, was extended here to a large majority of the dysregulated miRNAs. This pattern may reflect both the reduced muscle mass and physical activity of the older DMD patients (quantitative changes), as well pathophysiological evolution, independently of muscle mass and activity (qualitative changes). We confirmed the up-regulation of many miRNAs identified in previous investigations, including the dystromiRs, the cardiomiRs, 
and the DLK1-DIO3 clustered miRNAs. ${ }^{14,15,40}$ Of interest, we are reporting here, to our knowledge for the first time in $\mathrm{DMD}$, the dysregulation of many members of the Let- 7 and miR-320 families. Among the newly identified dysregulated miRNA in DMD plasma, we identified many miRNAs known to play diverse roles in muscle pathophysiology, including miR-128, ${ }^{23}$ miR-199a, ${ }^{24}$ miR-223, ${ }^{25}$ miR-486, ${ }^{26}$ and the miR$29^{41}$ and miR-30 28 family members. As in previous investigations, we identified a higher number of up-regulated rather than down-regulated circulating miRNAs in DMD. Of interest in the present study is the large number of down-regulated miRNAs in DMD. The down-regulation of circulating miRNA is not explained by the alteration of sarcolemma permeability. Thus, it is likely to result from transcriptional adaptation in dystrophic tissues. Of the down-regulated circulating miRNAs, we noticed in particular miR-342 and miR-185, both of which target the SREBP/mevalonate pathway and the synthesis of cholesterol. ${ }^{42}$ Additionally, we noted the down-regulation of the entire (five members) miR-320 family, of which the biological consequences in the context of muscular dystrophy are as yet unknown.

\section{A holistic bioinformatics analysis of microRNA dysregulation predicted perturbation of cholesterol metabolism}

The conventional method for the biological interpretation of miRNA dysregulation is based on the analysis of the consequences of miRNAs dysregulation. This interpretation can be carried out by a pathway enrichment analysis of mRNA targets for the dysregulated miRNAs. The miRNA target genes analysis predicted enrichment for the KEGG pathways of the proteoglycan ECM-receptor interaction, ${ }^{43}$ focal adhesion, ${ }^{44}$ fatty acid biosynthesis, ${ }^{45}$ as well as several signalling pathways: Hippo, ${ }^{46}$ PI3K-Akt, oestrogen, ${ }^{47}$ FoxO, ${ }^{48}$ and $\mathrm{ErbB},{ }^{49}$ all of which are known to be dysregulated in DMD. The dysregulation of these pathways is well-known in the field and thus supports the pertinence of our miRNA dysregulation results. However, the target genes analysis failed to produce novel hypotheses or an explanation for the DMD pathophysiology. Importantly, among the dysregulated miRNAs, we noticed some positioned inside mRNA transcripts that are known to be dysregulated in the dystrophic muscle in DMD. This observation supports a hypothesis that, in DMD, miRNAs and their host-gene might be coordinately dysregulated, and thus, miRNA dysregulation may indicate a host-gene dysregulation. Taking these considerations into account, we hypothesized that a biological interpretation approach for circulating miRNA that combines both target and host genes, which is described schematically in Supporting Information, Figure S8, may provide an improved interpretation of DMD pathophysiology. The IPA-based gene-interaction analysis predicted three dysregulated networks, all of which contain the term lipid metabolism. The SREBP-1 molecule was identified as being central to the merged network of dysregulation in DMD. Indeed, in agreement with the bioinformatics prediction, gene expression analysis confirmed the significant up-regulation of members of the SREBP pathway in the gastrocnemius and the diaphragm in the young $\mathrm{mdx}$ mouse, providing supporting experimental data for the hypothesis of SREBP pathway dysregulation.

\section{Treating mice by simvastatin}

In the short duration of only 3 weeks simvastatin treatment, we observed improved dystrophic parameters, including the reduction of diaphragm fibrosis, which is yet not highly pronounced at the young $\mathrm{mdx}$ mouse. Likely, treating older mdx mice for a longer period would result in a stronger anti-fibrotic effect, as previously reported. ${ }^{18}$ of note, Whitehead et al. suggested that the positive effect of simvastatin is cholesterol-independent. ${ }^{18}$ Our results support that HMGCR and the accumulation of cholesterol in the muscle are the direct therapeutic targets of simvastatin in DMD. This proposition is in agreement with earlier reports of lipid and particularly of cholesterol metabolism abnormalities in DMD. ${ }^{11,50-53}$ Of particular interest, Steen et al. reported the improved dystrophic parameters in an mdx mouse that overexpress the NPC1 gene, an accelerator of intracellular cholesterol trafficking, ${ }^{51}$ Milad et al. reported the correlation between increased plasma cholesterol and accentuated muscular dystrophy in the mdx mouse, ${ }^{53}$ and most recently White et al. reported the high prevalence of plasma lipid abnormalities in DMD patients, ${ }^{52}$ which together support that perturbation of cholesterol metabolism affects the DMD phenotype. Not only does our study concords with these previous findings, it also provides a molecular framework for this dysregulation. In summary, from the plasma samples of a large DMD cohort, the present study identified that perturbation of lipid metabolism, and in particular of cholesterol homeostasis, plays an important role in the pathophysiology of DMD. Thus, cholesterol metabolism is a potential therapeutic target in DMD.

\section{Author contribution}

F. A. and A. V. H. designed and performed experiments, analysed results, and wrote the paper. M. S. and L. Su. performed experiments and analysed results. G. C. performed bioinformatics analyses. L. Se. and T. V. designed experiments, managed the clinical trial, and analysed results. S. B. coordinated the clinical trial. I. R. and D. I. designed experiments, managed the project, and wrote the manuscript. 


\section{Acknowledgements}

We are grateful to the In vivo evaluation, Imaging and Histology services of Genethon. We are grateful to the Myobank, the tissue bank of the, AFM, and the Myology Institute (Paris, France) for providing DMD and healthy control skeletal muscle biopsies. This study was financially supported by the Association Francais contre les Myopathies (AFM); by ADNA (Advanced Diagnostics for New Therapeutic Approaches); the Institut National de la Sante et de la Recherche Medicale (INSERM); the Universite Pierre et Marie Curie Paris 06; the Centre National de la Recherche Scientifique (CNRS). The authors wish to thank patients and parents for participating, and all staff involved in sample collections in the different medical centres. The authors of this manuscript certify that they comply with the ethical guidelines for authorship and publishing in the Journal of Cachexia, Sarcopenia and Muscle.

\section{Online supplementary material}

Additional supporting information may be found online in the Supporting Information section at the end of the article.

Table S1. Cohort's medical center composition

Table S2. Clinical features of the DMD patients. Glucocorticoid treated $(T)$ patients are in blue. Untreated patients (UT) are in black. $\left({ }^{*}\right)$ from Martinique; $\left({ }^{* *}\right)$ Mother from the Reunion island and Martinique, Father Caucasian, $\left({ }^{* * *}\right)$ Mother from Brazil, Father Caucasian. NA: not available. ND: not done.

Table S3. Up-regulated plasma miRNA 4-12 years old DMD versus control. MiRNAs were ranked by FDR-adjusted $p$ value. MiRNA categories include dystromiRs, heart-enriched miRNAs (CardiomiR), miRNAs residing in the DLK1-DIO3 genomic locus, and Let-7 or miR-320 family members. MiRNAs of none of these categories were designed as unclassified.

Table S4. Down-regulated plasma miRNA 4-12 years old DMD versus control. MiRNAs were ranked by FDR-adjusted $p$ value. MiRNA categories include the miR-320 family members and unclassified miRNAs.

Table S5. dysregulation of glucocorticoid-responsive miRNAs in 4-12 years old DMD patients (5a, Table). Eleven DMD dysregulated miRNAs were GC-responsive. MiRNAs are classified by order of $p$-value in treated versus untreated DMD patients. All but miR-30e-5p and miR-425-5p were upregulated in DMD and reversed by the GC treatment toward normalization. In contrast, GC-treatment increased the downregulation in DMD of miR-30e-5p and miR-425-5p. Three miRNAs, miR-27-5p, miR-379-5p and let-7i-5p were no longer dysregulated after GC treatment. The miR-27-5p and miR-379-5p were not dysregulated in the group of all DMD patients versus healthy controls ( $p>0.05$ in red). $\mathrm{FC}=$ fold change, UT = untreated. (5b, Figure). Heat map of miRNA dysregulation. The miRNA relative level is illustrated by Z-scores, colored from blue to red, indicating from lowest to highest expression.

Table S6. Pathway enrichment analysis of target genes for dysregulated miRNAs, considering up- and down-regulated miRNAs in the 4-12 years old DMD/control samples (Adjusted p-value $<0.1$ ). Pathways are classified according to p-values (FDR-adjusted). Shown are the 20 most enriched pathways.

Table S7. List of the host genes of the identified dysregulated intragenic miRNAs that are known to be dysregulated in DMD models and patients. Both $3^{\prime}(3 p)$ and $5^{\prime}(5 p)$ mature miRNA forms of a given pre-miRNA were considered when both are dysregulated. The reference column refers to the study that described the dysregulation of the host gene.

Table S8. Dysregulated intragenic miRNA in DMD plasma and their related host-gene, considering all miRNAs embedded on the sense strand of introns and exons of protein-coding genes. FC and $p$ (student T-test) values are of miRNA expression in the blood plasma of 4-12 old DMD patients versus healthy controls. When both $3 p$ and $5 p$ isoforms of the same pre-miRNA were dysregulated, their host-genes were considered only once, with the lower $\mathrm{p}$-value miR isoform. Host genes for a number of different miRNAs were considered only once, taking into account FC and p-values of the miRNA with the lowest p-value.13

Table S9. RT-qPCR primers, and antibody information.

Figure S1. Venn diagram analysis of differentially expressed miRNAs in age and treatment stratified groups (a) Venn diagram of age-specific and age overlapping dysregulated miRNAs between DMD and healthy control samples and a table presentation of (a) is shown in (b). (c) Dysregulated miRNAs in the 4-20 years old. (d) Specific and overlapping dysregulated miRNAs in the 4-12 YO group between treated and untreated DMD versus healthy control. 34 and 49 miRNAs are dysregulated in the 4-8 and 8-12 years old group, respectively ( $a$ and $b$ ). No miRNA $(\mathrm{fdr}<0.1)$ is differentially-expressed between DMD and healthy control in the 12-20 YO group. When age groups are combined, 90 and 45 miRNAs are dysregulated in the 4-12 and 4-20 years old groups, respectively (a and b). Dysregulated miRNAs in the 4-12 years old DMD patients included all but one (miR-122) of the dysregulated miRNAs in the 4-20 years old group (c). When looking only at the 4-12 years old group, the classification into GC-treated and untreated as compared to the healthy control samples reduced the number of dysregulated miRNAs from 90 to 77 (d).

Figure S2. Correlogram of plasma miRNA expression in DMD patients of the 4-12 years old age group 
Expression correlations were analyzed among members of miRNA classes that were identified as dysregulated in the plasma in DMD patients. Dysregulated miRNA classes included the (a) MyomiRs, (b) CardiomiR, (c) Let-7 miRs, (d) miR-320 and (e) DLK1-DIO3 cluster miRNAs. Presented only significant Spearman correlations $(r<0.05)$, whereas " $X$ " represents the absence of correlation. The elliptic shape and the relative intensity of the blue color are proportional to the level of positive correlation. Correlation $r$ values are presented inside the elliptic shapes. The $r$ values for the correlations in the DLK1-DIO3 miRNAs (e) are presented in (f).

Figure S3. Cholesterol level in the serum of $\mathrm{mdx}$ mice treated by Simvastatin. Serum cholesterol in a 7-week old (young adult) $\mathrm{mdx}$ and control mice treated by Simvastatin during three weeks

Figure S4. Filipin staining of free cholesterol in $\mathrm{C} 2 \mathrm{C} 12$ cells C2C2 myoblast were treated by the cholesterol trafficking inhibitor U18666A (Lu et al., 2015), without (middle) or with (right) 24 hours treatment by the cholesterol synthesis inhibitor, simvastatin. Nucleus was stained by Syto13. Accumulated free cholesterol was stained with filipin.

Figure S5. Confocal analysis of cholesterol expression in transversal sections of the Tibialis anterior and the Gastrocnemius muscles

Transversal sections of $\mathrm{mdx}$ and control healthy $\mathrm{C} 57 \mathrm{BI} / 10$ mice, Gastrocnemius (GA) and Tibialis Anterior (TA), were labeled by Syto13 (nucleus) in blue, and Filipin (cholesterol) in red. Mdx mice were untreated (center; $\mathrm{mdx}$ ), or treated (right; $\mathrm{mdx}+$ Simv) by Simvastatin.

Figure S6. Confocal analysis of SREBP-2 expression in the mouse diaphragm

Transversal sections were immunostained with wheat germ agglutinin (WGA) in green, which stains the sarcolemma and the myonucleus membrane, with DAPI for nuclear staining, and with the anti-SREBP-2 antibody. White arrows indicate myonucleus in a central position in regenerated myofibres. Note that $\mathrm{mdx}$ nucleus are positive to all three colors (pink), while only residual faint red dotes can be observed in the nucleus (blue) of the $\mathrm{mdx}$ treated by simvastatin, demonstrating the downregulation of nuclear SREBP-2.
Figure S7. Effect of simvastatin on markers of oxidative stress (Nox2) and autophagic flux (LC3) in the dystrophic skeletal muscle

Western blotting analysis (upper panel) and its quantification (lower panel) of diaphragm biopsies of 10 weeks old mice $(n=6)$, treated or not (Simvastatin during three weeks, $80 \mu \mathrm{g} / \mathrm{kg}$, orally, integrated into the diet). The protein expression is normalized to GAPDH. All markers are upregulated in $\mathrm{mdx}$ compared to control mice. No significant differences observed in $\mathrm{mdx}$ mice upon simvastatin treatment

Figure S8. Approach for the biological interpretation of miRNA dysregulation, based on both target and host genes of the dysregulated miRNAs

Transcriptional adaptation in the diseased tissue affects both intergenic miRNAs (miRNA between genes, on the left) and intragenic miRNAs (miRNA inside genes, on the right). Intragenic miRNAs are often co-transcribed with their host genes, cooperatively affecting downstream events. The functional link between a miRNA to its host gene is thought to be strong, while the link between a miRNA to its many predicted target genes is questionable. Dysregulation of intragenic miRNAs and their host genes provides information on upstream signaling in the disease, which causes transcriptional dysregulation, while the analysis of target genes may provide information on downstream events, which are the consequences of miRNA dysregulation.

\section{Conflict of interest}

L. S. is member of the SAB or has performed consultancy for Sarepta, Dynacure, Santhera, Avexis, Biogen, Cytokinetics and Roche, Audentes Therapeutics and Affinia Therapeutics. T. V. is the Chief Scientific Officer of DiNAQOR AG. He also serves on the data safety monitoring board for trials sponsored by Italfarmaco and Sarepta. He is a consultant for Antisense Therapeutics, BioPhytis, Catabasis, Constant Therapeutics, Italfarmaco, Prosensa, Sarepta, Solid Biosciences and Syneos. All other authors declare no competing interest.

\section{References}

1. Guiraud S, Aartsma-Rus A, Vieira NM Davies KE, van Ommen G-JB, Kunkel LM. The pathogenesis and therapy of muscular dystrophies. Annu Rev Genomics Hum Genet 2015;16:281-308.

2. Ervasti JM, Campbell KP. A role for the dystrophin-glycoprotein complex as a transmembrane linker between laminin and actin. $J$ Cell Biol 1993;122:809-823.
3. Goemans N, Buyse G. Current treatment and management of dystrophinopathies. Curr Treat Options Neurol 2014;16:287.

4. Chamberlain JRJSJR. Chamberlain JRJSJR. American Society of Gene and Cell Therapy: Progress toward gene therapy for Duchenne MUSCULAR dystrophy; 2017.

5. Dalakas MC. Gene therapy for Duchenne muscular dystrophy: balancing good science, marginal efficacy, high emotions and excessive cost. SAGE Publications Ltd; 2017.

6. Randeree L, Eslick GD. Eteplirsen for paediatric patients with Duchenne muscular dystrophy: a pooled-analysis. J Clin Neurosci 2017;49:1-6.

7. Rodríguez-Cruz M, Sanchez R, Escobar RE, Cruz-Guzmán ODR, López-Alarcón $M$, Bernabe García M, et al. Evidence of insulin 
resistance and other metabolic alterations in boys with Duchenne or Becker muscular dystrophy. Int J Endocrinol 2015;2015:1-8.

8. Stapleton DI, Lau X, Flores M, Trieu J, Gehrig SM, Chee A, et al. Dysfunctional muscle and liver glycogen metabolism in mdx dystrophic mice. PLoS One 2014;9: e91514.

9. Amaral $A R$, Brunetto MA, Brólio MP, Cima DS, Miglino MA, Santos JPF, et al. Abnormal carbohydrate metabolism in a canine model for muscular dystrophy. I Nutr Sci 2017;6:e57.

10. Timpani CA, Hayes A, Rybalka E. Revisiting the dystrophin-ATP connection: how half a century of research still implicates mitochondrial dysfunction in duchenne muscular dystrophy aetiology. Med Hypotheses 2015;85:1021-1033.

11. Srivastava NK, Yadav R, Mukherjee S, Pal L, Sinha N. Abnormal lipid metabolism in skeletal muscle tissue of patients with muscular dystrophy: in vitro, high-resolution NMR spectroscopy based observation in early phase of the disease. Magn Reson Imaging 2017;38:163-173.

12. Cacchiarelli D, Legnini I, Martone J, Cazzella $V$, D'Amico A, Bertini E, et al. miRNAs as serum biomarkers for Duchenne muscular dystrophy. EMBO Mol Med 2011;3 258-265.

13. Zaharieva IT, Calissano $M$, Scoto $M$, Preston $\mathrm{M}$, Cirak S, Feng L, et al. Dystromirs as serum biomarkers for monitoring the disease severity in duchenne muscular dystrophy. PLoS One 2013;8:e80263.

14. Vignier $N$, Amor F, Fogel $P$, Duvallet $A$, Poupiot J, Charrier $S$, et al. Distinctive serum miRNA profile in mouse models of striated muscular pathologies. PLOS One 2013;8:e55281.

15. Jeanson-Leh L, Lameth J, Krimi S, Buisset J, Amor F, Le Guiner C, et al. Serum profiling identifies novel muscle miRNA and cardiomyopathy-related miRNA biomarkers in golden retriever muscular dystrophy dogs and Duchenne muscular dystrophy patients. Am J Pathol 2014;184: 2885-2898.

16. Li X, Li Y, Zhao L, Zhang D, Yao X, Zhang H, et al. Circulating muscle-specific miRNAs in Duchenne muscular dystrophy patients. Mol Ther Nucleic Acids 2014;3:e177.

17. Roberts TC, Blomberg KE, McClorey G, Andaloussi SE, Godfrey C, Betts C, et al. Expression analysis in multiple muscle groups and serum reveals complexity in the microRNA transcriptome of the $\mathrm{mdx}$ mouse with implications for therapy. $\mathrm{Mol}$ Ther Nucleic Acids 2012;1:e39.

18. Whitehead NP, Kim MJ, Bible KL, Adams $\mathrm{ME}$, Froehner SC. A new therapeutic effect of simvastatin revealed by functional improvement in muscular dystrophy. Proc Natl Acad Sci 2015;112 12864-12869.

19. Vlachos IS, Zagganas K, Paraskevopoulou $\mathrm{MD}$, Georgakilas G, Karagkouni D, Vergoulis $T$, et al. DIANA-miRPath v3.0: deciphering microRNA function with experimental support. Nucleic Acids Res 2015;43: W460-W466.
20. Hinske LC, Franca GS, Torres HAM, Ohara DT, Lopes-Ramos CM, Heyn J, et al. miRIAD--integrating microRNA inter- and intragenic data. Database 2014;2014: bau099-bau099.

21. Yu G, He QY. ReactomePA: an R/ Bioconductor package for reactome pathway analysis and visualization. Mol Biosyst 2016;12:477-479.

22. Kozomara A, Griffiths-Jones S. miRBase: annotating high confidence microRNAs using deep sequencing data. Nucleic Acids Res 2014;42:D68-D73

23. Motohashi N, Alexander MS, ShimizuMotohashi Y, Myers JA, Kawahara G, Kunke LM. Regulation of IRS1/Akt insulin signaling by microRNA-128a during myogenesis. Cell Sci 2013;126:2678-2691.

24. Alexander MS, Kawahara G, Motohashi N, Casar JC, Eisenberg I, Myers JA, et al. MicroRNA-199a is induced in dystrophic muscle and affects WNT signaling, cell proliferation, and myogenic differentiation. Cell Death Differ 2013;1-15.

25. Greco $S$, De Simone M, Colussi C, Zaccagnini G, Fasanaro $P$, Pescatori $M$, et al. Common micro-RNA signature in skeletal muscle damage and regeneration induced by Duchenne muscular dystrophy and acute ischemia. FASEB J 2009;23:3335-3346.

26. Small EM, O'Rourke JR, Moresi V, Sutherland LB, McAnally J, Gerard RD, et al. Regulation of PI3-kinase/Akt signaling by muscle-enriched microRNA-486. Proc Natl Acad Sci U S A 2010;107:4218-4223.

27. Li J, Chan MC, Yu Y, Bei Y, Chen P, Zhou $Q$ et al. miR-29b contributes to multiple types of muscle atrophy. Nat Commun 2017;8:15201.

28. Guess MG, Barthel KKB, Harrison BC Leinwand LA. miR-30 family microRNAs regulate myogenic differentiation and provide negative feedback on the microRNA pathway. PLoS One 2015;10: e0118229.

29. Fiorillo $A A$, Heier $C R$, Novak JS, Tully $C B$, Brown KJ, Uaesoontrachoon K, et al. TNF$\alpha$-induced microRNAs control dystrophin expression in Becker muscular dystrophy. Cell Rep 2015;12:1678-1690.

30. Baskerville S, Bartel DP. Microarray profiling of microRNAs reveals frequent coexpression with neighboring miRNAs and host genes. RNA 2005;11:241-247.

31. Hinske L, Galante PA, Kuo WP, OhnoMachado L. A potential role for intragenic miRNAs on their hosts' interactome. BMC Genomics 2010;11:533.

32. Boivin V, Deschamps-Francoeur G, Scott MS. Protein coding genes as hosts for noncoding RNA expression. Semin Cell Dev Biol 2018;75:3-12.

33. Wang $Y X$, Feige $P$, Brun $C E$, Hekmatnejad $B$, Dumont NA, Renaud J-M, et al. EGFR-Aurka signaling rescues polarity and regeneration defects in dystrophin-deficient muscle stem cells by increasing asymmetric divisions. Cell Stem Cell 2019;24:419-432.e6.

34. Dorchies OM, Reutenauer-Patte J, Dahmane E, Ismail HM, Petermann O, Patthey-Vuadens $\mathrm{O}$, et al. The anticancer drug tamoxifen counteracts the pathology in a mouse model of Duchenne muscular dystrophy. Am J Pathol 2013;182:485-504.

35. Brown MS, Goldstein JL. The SREBP pathway: regulation of cholesterol metabolism by proteolysis of a membrane-bound transcription factor. Cell 1997;89:331-340.

36. Yang $T$, Espenshade PJ, Wright ME, Yabe $D$, Gong $Y$, Aebersold R, et al. Crucial step in cholesterol homeostasis: sterols promote binding of SCAP to INSIG-1, a membrane protein that facilitates retention of SREBPS in ER. Cell 2002;110:489-500.

37. Shimano H, Sato R. SREBP-regulated lipid metabolism: convergent physiology-divergent pathophysiology. Nat Rev Endocrinol 2017;13:710-730.

38. Rouillon J, Poupiot J, Zocevic A, Amor F, Léger $\mathrm{T}$, Garcia $\mathrm{C}$, et al. Serum proteomic profiling reveals fragments of MYOM3 as potential biomarkers for monitoring the outcome of therapeutic interventions in muscular dystrophies. Hum Mol Genet 2015;24:4916-4932.

39. Israeli D, Poupiot J, Amor F, Charton K, Lostal W, Jeanson-Leh $\mathrm{L}$, et al. Circulating miRNAs are generic and versatile therapeutic monitoring biomarkers in muscular dystrophies. Sci Rep 2016;6:28097.

40. Sanson M, Hog Vu A, Massourides E, Bourg $\mathrm{N}$, Suel L, Amor F, et al. miR-379 links glucocorticoid treatment with mitochondrial response in Duchenne muscular dystrophy. Sci Rep 2020;10:9139.

41. Zanotti S, Gibertini S, Curcio M, Savadori $P$, Pasanisi B, Morandi L, et al. Opposing roles of miR-21 and miR-29 in the progression of fibrosis in Duchenne muscular dystrophy. Biochim Biophys Acta 1852;2015: 1451-1464.

42. Li X, Chen YT, Josson S, Mukhopadhyay NK, Kim J, Freeman MR, et al. MicroRNA-185 and 342 inhibit tumorigenicity and induce apoptosis through blockade of the SREBP metabolic pathway in prostate cancer cells. PLoS One 2013;8:e70987.

43. Carmen L, Maria V, Morales-Medina JC, Vallelunga A, Palmieri B, lannitti $T$. Role of proteoglycans and glycosaminoglycans in Duchenne muscular dystrophy. Glycobiology 2019;29:110-123.

44. Burkin DJ, Wallace GQ, Nicol KJ, Kaufman DJ, Kaufman SJ. Enhanced expression of the $\alpha 7 \beta 1$ integrin reduces muscular dystrophy and restores viability in dystrophic mice. J Cell Biol 2001;152:1207-1218.

45. Le Borgne F, Guyot S, Logerot M, Beney L, Gervais $P$, Demarquoy J. Exploration of lipid metabolism in relation with plasma membrane properties of Duchenne muscular dystrophy cells: influence of L-carnitine. PLoS One 2012;7:e49346.

46. Vita GL, Polito F, Oteri R, Arrigo R, Ciranni AM, Musumeci $O$, et al. Hippo signaling pathway is altered in Duchenne muscular dystrophy. PLoS One 2018;13:e0205514.

47. Maani N, Sabha N, Rezai K, Ramani A Groom L, Eltayeb N, et al. Tamoxifen therapy in a murine model of myotubular myopathy. Nat Commun 2018:9:4849. 
48. Parolo S, Marchetti L, Lauria M, Misselbeck K, Scott-Boyer M-P, Caberlotto $L$, et al. Combined use of protein biomarkers and network analysis unveils deregulated regulatory circuits in Duchenne muscular dystrophy. PLoS One 2018;13:e0194225.

49. Handschin C, Kobayashi YM, Chin S, Seale P, Campbell KP, Spiegelman BM. PGC-1 $\alpha$ regulates the neuromuscular junction program and ameliorates Duchenne muscular dystrophy. Genes Dev 2007;21:770-783.
50. Strakova J, Kamdar F, Kulhanek D, Razzoli M, Garry DJ, Ervasti JM, et al. Integrative effects of dystrophin loss on metabolic function of the $\mathrm{mdx}$ mouse. Sci Rep 2018;8:13624.

51. Steen MS, Adams ME, Tesch Y, Froehner SC. Amelioration of muscular dystrophy by transgenic expression of niemann-pick C1. Mol Biol Cell 2009;20:146-152.

52. White $Z$, Hakim $\mathrm{CH}$, Theret $\mathrm{M}$, Yang $\mathrm{NN}$, Rossi F, Cox D, et al. High prevalence of plasma lipid abnormalities in human and canine Duchenne and Becker muscular dystrophies depicts a new type of primary genetic dyslipidemia. J Clin Lipidol 2020;14: 459-469.e0.

53. Milad N, White Z, Tehrani AY, Sellers S, Rossi FMV, Bernatchez P. Increased plasma lipid levels exacerbate muscle pathology in the mdx mouse model of Duchenne muscular dystrophy. Skelet Muscle 2017;7:19. 